\title{
Pulmonary Embolism in Patients with Covid-19 in Direct Oral Anticoagulant
}

\author{
Fabio Augusto De Luca, ${ }^{\circledR}$ Guilherme Arruda, ${ }^{1}$ Vinicius Esteves, ${ }^{1}$ André Feldman, ${ }^{1 \odot}$ Ítalo Bruno dos Santos \\ Sousa, ${ }^{\circledR \oplus}$ José Célio Vieira Brandão, ${ }^{1 \oplus}$ Raquel Muarrek Garcia, ${ }^{1 \oplus}$ Olga Ferreira de Souza ${ }^{\circledR}$ \\ Rede D'Or São Luiz,' São Paulo, SP - Brazil \\ Universidade de São Paulo, INCOR,2 São Paulo, SP - Brazil
}

\section{Introduction}

The global pandemic caused by the new coronavirus (COVID-19) has been promoting major challenges and unprecedented clinical decisions in the medical scenario. Currently, according to the World Health Organization, the disease affects over 185 countries, with over 12 million people infected and nearly 500 thousand deaths. ${ }^{1}$

Among the most serious manifestations of the syndrome, the high prevalence of thromboembolic events, up to $31 \%$ of the patients hospitalized in intensive care units, according to an international series, calls attention. ${ }^{2-4}$ Different mechanisms of action related to the infection by SARS-CoV-2 may be related to thrombosis: excessive inflammation, tissue hypoxia, immobilization, and disseminated intravascular coagulation (DIC); however, one question remains: Is the etiology linked to some specific effect of the virus or a reflex of the severity of the case ${ }^{5}$ Despite the anticoagulant therapy emerging as a major weapon in the therapeutic arsenal, data is still scarce in the literature regarding the efficacy and safety of warfarin or direct oral anticoagulants (DOAC) in this specific setting. ${ }^{6}$ It should be highlighted that, of the 1.5 million people currently infected in Brazil, ${ }^{1}$ a significant number present a history of cardiovascular disease and are presently taking anticoagulants. This class of drugs may show serious interactions with investigational therapies currently employed for COVID-19, which could increase the risk of thrombotic or hemorrhagic events. In this context, it becomes imperative to highlight

\section{Keywords}

COVID-19; Pandemics; Respiratory Acute Syndrome; Pulmonary Embolism; Anticoagulants. that the thrombosis associated with COVID-19 and anticoagulant therapy is a unique scenario and must be investigated.

In this light, the report below presents the case of a patient diagnosed with COVID-19 with a medical history of chronic atrial fibrillation (CAF) and who, despite his regular use of DOACs, has evolved to pulmonary embolism and deep venous thrombosis.

\section{Case Report}

A male, 46-year-old Japanese patient was admitted to the emergency room of the hospital, reporting a condition of non-specific malaise, associated with fever peaks and body aches for 3 days. The patient presented a medical history of obesity (BMI- $38.1 \mathrm{~kg} / \mathrm{m}^{2}$ ); systemic high blood pressure, treated with losartan, $50 \mathrm{mg} /$ day; diabetes mellitus, treated with metformin, $1000 \mathrm{mg} /$ day; and a diagnosis of atrial fibrillation for approximately 3 years, treated with apixaban, $5 \mathrm{mg}$ twice a day, and metoprolol, $50 \mathrm{mg}$ twice a day. Physical examination upon hospital admission showed: BP: 130 x70 mmHg, HR: 93bpm, O2 Sat: 94\% Axillary temperature: 37.8 degrees (despite using antipyretic medication). The patient also presented hyperemic oropharynx, but with no secretion, a moderate pain upon palpation of the facial sinuses, and cardiorespiratory auscultation with no significant change. The Padua score is meant to risk stratify patients who have a potential risk for VTE. The Padua Score has 11 baseline features, and patients $\geq 4$ tend to present a high risk of VTE. In this case, the PADUA score was 5 (decreased mobility-3, obesity-1, active infection-1).

The admission electrocardiogram (ECG) confirmed the diagnosis of atrial fibrillation and diffuse changes of ventricular repolarization (Figure 1) 
Figure 1 - Admission ECG with atrial fibrillation rhythm and diffuse change of repolarization.

Initial lab tests revealed leukopenia and thrombocytopenia, and after 48 hours, a D-dimer increase of almost 3 times the baseline value and maintenance of thrombocytopenia was idenitified (Figure 2).

The chest computed tomography (CT) imaging showed ground-glass opacities and bilateral consolidation foci, predominant in peripheral regions (Figure 3A), estimating a $25 \%$ to $50 \%$-involvement of the pulmonary parenchyma. Due to the patient's clinical condition, together with the tomographic imaging suggesting viral pneumonia, the patient was started on Oseltamivir, venous hydration, and symptomatic care. The next day after hospitalization, the RT-PCR for COVID-19 was confirmed, and hydroxichloroquine $(800 \mathrm{mg}$ on the first day, followed by $400 \mathrm{mg}$ on subsequent days), associated with clarithromycin (500 mg twice a day) was administered. Due to atrial fibrillation with a high ventricular response (FC around $130 \mathrm{bpm}$ ), the resumption of metoprolol, $50 \mathrm{mg}$ twice a day, was chosen. On the fifth day of hospitalization (eight days after the onset of symptoms), a significant worsening was observed in the patient's respiratory condition, with a worsening of saturation and tachypnea; as a result, methylprednisolone, $40 \mathrm{mg}$ twice a day, was introduced. Concurrently to the clinical deterioration, a significant increase in D-dimer (above 10,000 ng/ml) was also observed. Therefore, the decision was made to perform a chest $\mathrm{CT}$, respecting the protocol for pulmonary embolism (Figure 3B), and the diagnosis of pulmonary thromboembolism (PTE) was confirmed, with bilateral segmental and subsegmental involvement. Antibiotic spectrum was expanded to Tazobactam and Linezolide due to the worsening in the radiological imaging and suspected bacterial association. Subsequently, a combination of lopinavir and ritonavir was started.
The anticoagulant scheme was changed with the interruption of apixaban and the start of intravenous unfractioned heparin with aPTT control (target 1.5 to 2.5 times the normal value). Echocardiography showed satisfactory ventricular performance: left ventricle ejection fraction of $58 \%$, pulmonary artery pressure: 35 $\mathrm{mmHg}$, and no right ventricle impairment. No troponin increase was observed; however, there was a BNP peak of 231 (TN $100 \mathrm{pg} / \mathrm{ml}$ ). Deep venous thrombosis was observed in the left gastrocnemius vein, identified in the lower limb doppler.

After the institution of therapy and intensive support care, the patient experienced stabilization and posterior gradual improvement of the respiratory and infectious condition, with the normalization of lab tests, and after 15 days of hospitalization, the patient was discharged and medicated with rivaroxaban, $15 \mathrm{mg}$ twice a day for a 21day period. After this period, the dose was changed to 20 mg once a day. At Day 30 follow-up, the patient returned to the outpatient clinic in good clinical condition, with no complaints, maintaining outpatient follow-up with the cardiologist and infectologist.

\section{Discussion}

Since the beginning of the course of the COVID-19 pandemic in Brazil, on February 26, 2020, some international studies were already highlighting different cases of pulmonary embolisms in patients with COVID-19 and a general incidence of around $23 \%$ to $31 \% .^{7-9}$

In a survey of 328 patients who had a positive RTPCR, 22\% were diagnosed with pulmonary embolism. In this sample, patients with a body mass index above $30 \mathrm{~kg} / \mathrm{m} 2$ were more frequently diagnosed (58\% vs. 
Table 1 - Laboratory test results

\begin{tabular}{|c|c|c|c|c|c|c|c|}
\hline Test & $03 / 24$ & $03 / 25$ & $03 / 26$ & $03 / 27$ & $03 / 28$ & $03 / 29$ & $03 / 30$ \\
\hline Hemoglobin (g/dL) & 16.2 & 15.0 & 14.5 & 15.2 & & 14.8 & 14.9 \\
\hline Leukocytes $/ \mathrm{mm}^{3}$ & 3,080 & 3,340 & 2,960 & 3,800 & & 5,240 & 7,390 \\
\hline Platelets $/ \mathrm{mm}^{3}$ & 68,000 & 74,000 & 73,000 & 86,000 & & 94,000 & 115,000 \\
\hline D-dimer (ng/mL) & 408 & 1041 & 1116 & 1702 & 4596 & $>10,000$ & \\
\hline Ferritin (mg/dL) & 3576 & 4646 & 4170 & 4774 & 3603 & & 3384 \\
\hline Troponin (ng/mL) & $<0.16$ & & & $<0.16$ & & & \\
\hline $\mathrm{BNP}(\mathrm{pg} / \mathrm{mL})$ & & & & & 174 & & \\
\hline PCR (mg/dL) & 3.0 & 3.88 & 5.53 & 7.65 & 4.48 & 10.6 & \\
\hline Creatinine (mg/dL) & 0.84 & 0.89 & 0.79 & & & 0.75 & 0.85 \\
\hline Arterial $\mathrm{pH}$ & & & & 7.44 & & 7.47 & \\
\hline Arterial pO2 (mmHg) & & & & 80 & & 63 & \\
\hline Lactate (mg/dL) & 11.3 & 20.4 & & 25.3 & & & \\
\hline Test & $03 / 31$ & $04 / 01$ & $04 / 02$ & $04 / 03$ & $04 / 04$ & $04 / 05$ & $04 / 06$ \\
\hline Hemoglobin (g/dL) & 14.7 & & 14.5 & & 15.3 & 15.1 & 14.0 \\
\hline Leukocytes $/ \mathrm{mm}^{3}$ & 8,210 & & 8,500 & & 8,980 & 9,450 & 8,540 \\
\hline Platelets $/ \mathrm{mm}^{3}$ & 99,000 & & 130,000 & & 164,000 & 171,000 & 159,000 \\
\hline D-dimer (ng/mL) & $>10,000$ & $>10,000$ & $>10,000$ & 5285 & 3958 & 3465 & 1712 \\
\hline Ferritin (mg/dL) & 783 & 3675 & 3382 & 3425 & & & \\
\hline Troponin (ng/mL) & $<0.16$ & & $<0.16$ & & & $<0.16$ & $<0.16$ \\
\hline BNP (pg/mL) & & & 231 & 99 & & & \\
\hline PCR (mg/dL) & 2.73 & 1.56 & 1.24 & 1.69 & 1.23 & 0.46 & 0.25 \\
\hline Creatinine (mg/dL) & & & 0.87 & 0.84 & 0.95 & & \\
\hline Arterial pH & & & & 7.48 & 7.46 & 7.46 & \\
\hline Arterial pO2 (mmHg) & & & & 60 & 75 & 72 & \\
\hline Lactate (mg/dL) & & & 2.3 & & & 38.6 & \\
\hline
\end{tabular}

$44 \%)$. Both the mean D-dimer was $(9.33 \mathrm{mcg} / \mathrm{ml}$ vs 2.54 $\mathrm{mcg} / \mathrm{ml}, \mathrm{p}=0.001$ ) as well as oxygen supplementation were higher in this group diagnosed with pulmonary embolism (4.3 vs 2.7, p=0.007). Regarding the location of the thrombi, $51 \%$ are segmental, 31\% lobar, $13 \%$ central, and $5.5 \%$ subsegmental. ${ }^{8}$

The case reported here follows a profile of patients at higher risk due to obesity and an increase in dimer. Moreover, the CT scan is in accordance with that found with a higher prevalence in the literature, that of segmental and subsegmental involvement.

The presence of pulmonary embolism and venous thromboembolism is a prognostic marker, increasing mortality by 3.3 fold. This fact increases the discussion of the early use of anticoagulants in these patients. D-dimer variation has been considered to be one of the possibilities for therapeutic recommendation; however, there is still no general consensus. ${ }^{10,11}$ In this case, after the diagnosis 

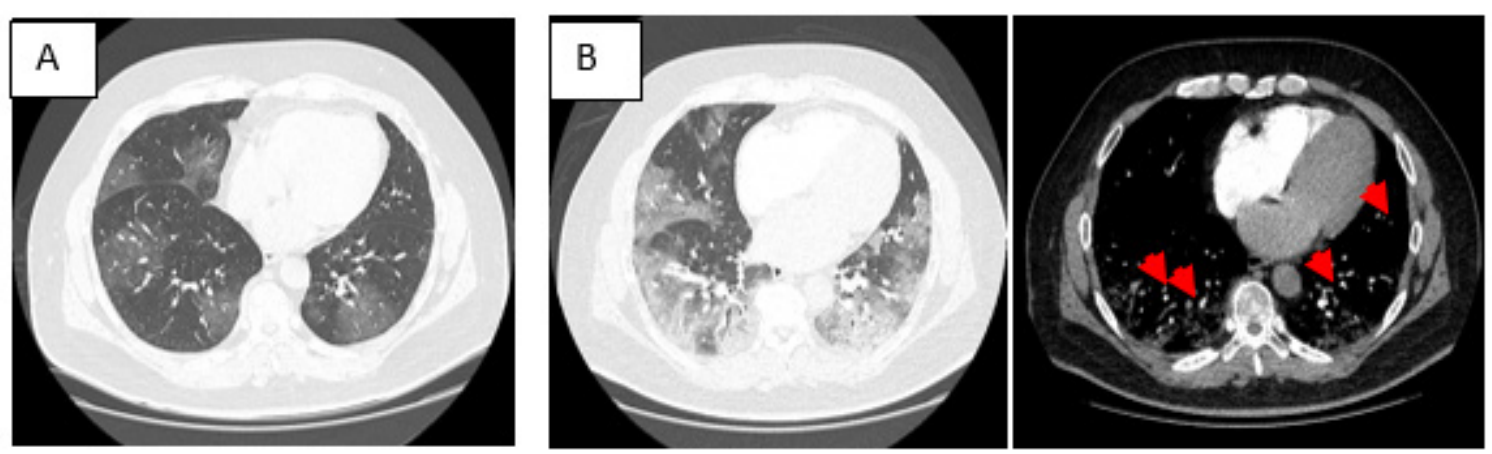

Figure 2-A) Admission chest CT with ground-glass opacities B) Chest CT on the fifth day of hospitalization with worsening of ground-glass imaging pattern and diagnostic angiotomography for pulmonary embolism

of an embolism, the patient experienced a significant worsening in his general health and was admitted to the ICU on oxygen supplementation.

Regarding the prophylaxis of thrombotic events, a retrospective study at the Tongji hospital, in WuhanChina, 449 patients with a severe course of COVID-19, only 99 (22\%) received heparin (both low molecular weight and unfractionated) for 7 days or longer. The 28-day mortality was higher in the group not receiving anticoagulants ${ }^{12-14}$ The patient was initially managed with the maintenance of DOACs, as he was already taking apixaban, at the therapeutic dose, to prevent embolic events during atrial fibrillation. However, after the confirmation of the pulmonary embolism and venous thrombosis of the lower limbs, apixaban was interrupted, and unfractionated heparin was started, with seeking to promote a lab control of anticoagulation through aPTT and to produce the potential benefit of pleiotropic actions ascribed to heparin, primarily due to the description, in COVID-19, of the action of proinflammatory cytokines, such as: IL-2, IL6, IL-7, IL-10, G-CSF, IP-10, MCP-1, MIP- $1^{\text {a }}$, and TNF- $\alpha .{ }^{15}$ Another key factor associated with the trend of replacing DOACs by heparin is that this procedure showed a change in DOAC blood levels with the concurrent use of antivirals, such as lopinavir/ritonavir. ${ }^{16}$

The option to use rivaroxaban upon hospital discharge was due to the proof of efficacy and safety shown in Einstein-PE and Einstein-VTE ${ }^{16,17}$ studies, with a higher dose in the first 21 days (15 mg twice a day), followed by $20 \mathrm{mg}$ on an ongoing basis due to the presence of atrial fibrillation. ${ }^{18}$ The logic associated with the higher initial dose was considered beneficial, as it could enhance the anticoagulant action in the initial post-COVID-19 recovery phase, a regression point of the inflammatory condition but still of unknown pro-thrombotic extension.

Even though DOACs have been approved for the treatment of pulmonary embolism and VTE prevention in the general population, their efficacy in this specific situation is still unknown and thus impossible to affirm. It should be noted that the mechanism of thrombosis is still relatively unknown and may well be related to the activation of multiple coagulation pathways, which can lead to the assumption that DOAC does not in fact provide full coverage.

It is also important to highlight that, even when there is no thrombotic event, as in the case reported here, some societies have been adopting a favorable position regarding continuous prophylactic anticoagulation. In a recent publication, the Italian Society on Thrombosis and Hemostasis (SISET), suggests the maintenance of the extended prophylaxis at home for 7-14 days after discharge. This Italian guideline also reinforces that patients with therapeutic doses of heparin or DOAC must have the renal function, as well as an anti-Xa factor, ${ }^{15}$ monitored.

\section{Conclusion}

Pulmonary embolism is a frequent complication in patients diagnosed with COVID-19 and is a severity marker, highlighting the pro-thrombotic status present in this illness. Patients with cardiovascular disease, who are treated with the chronic use of antiplatelets and oral anticoagulants, makes it difficult to define anticoagulation during the period of a thrombotic 
storm imposed by the Sars-Cov-2 infection. Many questions related to anticoagulation, thrombotic events, and COVID-19 still remain. While we wait for the randomized studies promoting such answers, the guidance based on the already established anticoagulation guidelines and on the individualization of the benefit and risk associated with each patient must guide the treatment in such cases.

\section{Author Contributions}

Acquisition of data: De Luca FA, Arruda G, Sousa IB, Brandao JC, Garcia RM. Conception and design of the research: De Luca FA, Arruda G, Esteves V, Souza O, Feldman A. Analysis and interpretation of the data: De Luca FA, Arruda G, Esteves V, Souza O, Feldman A. Writing of the manuscript: De Luca FA, Arruda G, Sousa IB.

\section{References}

1. World Health Organizaton. (WHO) Coronavirus Disease (COVID-19) Dashboard. [Cited in 2020 April 12] Available from: https://covid19.who. int/?gclid=

2. Klok FA,Kruip MJ, van der Mees NJ, Arbous MS, Gommers DA, Kant $\mathrm{KM}$, Kaptein FH, et al. Incidence of thrombotic complications in critically ill ICU patients with COVID-19. Thromb Res. 202);191:145-7.

3. Cui S, Chen S, Li X, Liu S, Wang F. Prevalence of venous thromboembolism in patients with severe novel coronavirus pneumonia. J Thromb Haemost. 2020;18(6):1421-4

4. Helms J,Tacquard C, Severac F, Lorant IL, Ohana G, Delabrunche $\mathrm{X}$, et al. High risk of thrombosis in patients in severe SARS-CoV-2 infection: a multicenter prospective cohort study. Intensive Care Medicine.2020;46(6):1089-98

5. Bikdeli B, Madhavan MV, Jimenez D, Chuich T, Dreyfus I, Driggin E, et al. COVID-19 and thrombotic or thromboembolic disease: implications for prevention, antithrombotic therapy, and follow-up. J Am Coll Cardiol. 2020;75(23):2950-73.

6. Connors JM, Levy JH. COVID-19 and its implications for thrombosis and anticoagulation. Blood (2020) 135 (23): 2033-2040. https://doi.org/10.1182/ blood.2020006000

7. Danzi GB, Loffi M, Galeazzi G, Gherbesi E. Acute Pulmonary Embolism and COVID-19 Pneumonia: A Random Association? Eur Heart J. 2020; 41(19):1858.

8. Poyiadji N, Cormier P, Patel PY, Hadied MO, Bhagava P, Khanna K, et al. Acute pulmonar embolism and COVID-19. Radiology. 2020 May $14 ; 201955$.

9. Leonard-Lorant I,Delabranche X, Severac F, Helms J, Pauzet C, Collange O, et al. Acute Pulmonary Embolism in COVID-19 Patients on CT Angiography and Relationship to D-Dimer Levels. Radiology. April 2020. Grillet F, Behr J, Calame P, Aubry S, Delabrousse E. Acute
Critical revision of the manuscript for intellectual content: De Luca FA, Arruda G, Esteves V, Souza O, Feldman A.

\section{Potential Conflict of Interest}

No potential conflict of interest relevant to this article was reported.

\section{Sources of Funding}

There were no external funding sources for this study.

\section{Study Association}

This study is not associated with any thesis or dissertation work.

\section{Ethics Approval and Consent to Participate}

This article does not contain any studies with human participants or animals performed by any of the authors.

Pulmonary Embolism Associated with COVID-19 Pneumonia Detected by Pulmonary CT Angiography.Radiology. April 2020;296(3):E189-E191.

10. Harenberg J, Favaloro E. COVID_19: progression of disease and intravascular coagulation - present status and future perspectives. Clin Chem Lab Med. 2020;58(7):1029-36.

11. Middeldorp S, Coppens M, van Haaps TF, Foppen M, Vlaar AP, Muller $\mathrm{MC}$, et al. Incidence of venous thromboembolism in hospitalized patients with COVID-19. J Thromb Hemost.2020;18(8):1995-2002.

12. Porfidia A, Pola R. Venous thromboembolism and heparin use in COVID-19 patients: juggling between pragmatic choices, suggestions of medical societies. J Thromb Thrombolysis. 2020 ;50(1):68-71.

13. Thachil J, Tang N, Gando S, Falanga A, Cattaneo M, Levi M, Clark C, Iba T STH interim guidance on recognition and management of coagulopathy in COVID-19. J Thromb Haemost. 2020 May; 18(5):1023-6.

14. Zhou F, Yu T, Du R, Fan G, Liu Y, Liu Z, et al. Clinical course and risk factors for mortality of adult inpatients with COVID-19 in Wuhan, China: a retrospective cohort study. Lancet. 2020 Mar 28; 395(10229):1054-62.

15. Marietta M, Ageno W, Artoni A, De Candia E, Gresele P, Marchetti M, et al. COVID-19 and haemostasis: a position paper from Italian Society on Thrombosis and Haemostasis (SISET) Blood Transfus. 2020;18(3):167-9.

16. Testa, S, Prandoni, P, Paoletti, O, Morandini R, Tala M, Dellanoce C, et al. Direct oral anticoagulant plasma levels' striking increase in severe COVID-19 respiratory syndrome patients treated with antiviral agents: The Cremona experience. J Thromb Haemost. 2020; 18(6):1320-3.

17. auersachs R, Berkowitz SD, Brenner B,Buller H, Decousus H, Gallus AS, et al. Oral rivaroxaban for symptomatic venous thromboembolism. N Engl J Med. 2010; 363(26):2499-510.

18. Buller HR, Prins MH, Lensin AW,Decousus H, Jacobson BF, Minar E, et al. Einstein Investigators. Oral rivaroxaban for the treatment of symptomatic pulmonary embolism. N. Engl. J. Med. 2012;366(14):1287-97. 